\title{
Colaboradores satisfechos - productividad empresarial
}

\author{
Satisfied employees - business productivity \\ Magda Gabriela Sánchez-Trujillo ${ }^{a}$, Jorge Martín Hernández Mendoza ${ }^{b}$, \\ Héctor Daniel Molina Ruiz ${ }^{c}$, Ma. Lourdes E. García Vargas ${ }^{d}$
}

\begin{abstract}
:
The main challenge of an organization is the economic goal, which is to maximize business profit, researchers of the subject of job satisfaction measurement techniques identify and consider it an important tool for organizations seeking greater productivity and a significant contribution to human resources and the company.

Because of these needs, organizations are interested in understanding the concept of job satisfaction and the benefits it can bring to your business in areas such as productivity.

Therefore, the aim is to identify the degree to which workers are satisfied and identified with the organization and its impact on productivity, as companies seek to generate higher performance and benefits. The results prove theoretical elements and their respective indicators, comparing the issue of job satisfaction influence on productivity, in three medium-sized textile manufacturing located in the municipality of Tepeji del Rio, in Hidalgo State.
\end{abstract}

Keywords:

Job satisfaction, productivity, enterprise

\section{Resumen:}

El principal reto de una organización es el objetivo económico, que consiste en maximizar el beneficio empresarial, estudiosos del tema de satisfacción laboral identifican técnicas de medición y consideran que es una herramienta importante para las organizaciones que buscan mayor productividad y un aporte significativo al recurso humano y la empresa.

Debido a estas necesidades, las organizaciones se han interesado en entender el concepto de satisfacción laboral y los beneficios que pueden traer a su negocio en aspectos como la productividad.

Por tanto, se pretende identificar el grado en que los trabajadores están satisfechos e identificados con la organización y su impacto en la productividad, ya que las empresas buscan generar mayor rendimiento y beneficios. Los resultados prueban elementos teóricos y sus respectivos indicadores, que comparan el tema de influencia de satisfacción laboral en la productividad, en tres empresas medianas del sector manufacturero textil localizadas en el municipio de Tepeji del Rio, del Estado de Hidalgo.

Palabras Clave:

Satisfacción laboral, productividad, empresa

\section{Introducción}

La satisfacción laboral en el trabajo se basa en tener trabajadores satisfechos, en donde se tenga en cuenta las relaciones personales, la comunicación y el desarrollo de los líderes que se preocupen por conocer las motivaciones y necesidades de sus colaboradores enfocados a comprenderde qué forma se pueden utilizar en beneficio de ellos y de la organización.

La satisfacción laboral también es una tarea y responsabilidad del trabajador, ya que las empresas pueden hacer mucho por el bienestar de los trabajadores y brindarles herramientas de trabajo, espacios, beneficios sociales, etc., pero depende del trabajador y de la forma

\footnotetext{
a Autor de Correspondencia, Universidad Autónoma del Estado de Hidalgo, Escuela Superior Tepeji del Rio. https://orcid.org/-0000-00029093-1081,Email: magdags@uaeh.edu.mx.sitioweb@uaeh.edu.mx 
y responsabilidad como lo asuma y aproveche dentro de la compañía, (Ramirez, 2014).

Por otro lado, la productividad es un concepto que por necesidad se asocia a un producto obtenido pormedio de recursos utilizados para poder lograrlo, siendo posible cuantificar el desempeño de productividad en diferentes niveles de agregación. Se mide a nivel producto específico en términos físicos o por unidad de insumo: ejemplo toneladas de materia por hora empleada en la producción, (Hernández Laos, 2002).

Estudios de la Universidad de Warwick en Reino Unido, mencionan que la satisfacción laboral hace que la gente sea un $12 \%$ más productiva, al igual estudios de compañías como Google han invertido en apoyo para sus trabajadores, aumentando su satisfacción en un $37 \%$, ya que es reconocido que mostrar respeto y confianza hacia los trabajadores, aumenta sus niveles de felicidad y a su vez sus niveles de productividad y creatividad, (Andrew, 2010).

Una empresa con colaboradores satisfechos, es un lugar con mayor compromiso y productividad donde los mejores resultados se dan por sí solos gracias al compromiso de los trabajadores tal como afirma el autor (Shawn Achor), en su obra The happiness advantage*. Los trabajadores felices obtienen mayores niveles de productividad, venden más y actúan mejor en posiciones de liderazgo y obtienen un nivel salarial alto, gozan también de una mayor seguridad en el trabajo, logrando que los trabajadores puedan sustentar relaciones con efecto de sentirse satisfechos con los que aportan a la empresa, lo que impacta en un clima laboral estable que facilita alcanzar mayores desempeños.

De acuerdo con el cálculo de los índices de productividad de INEGI 2015, se menciona que la productividad es una medida de la eficiencia utilizada para combinar los factores productivos y los insumos para producir una determinada cantidad de bienes y servicios. Un incremento de la productividad implica producir más con la misma cantidad de recursos para producir el mismo volumen del producto.

El problema de la productividad es que no hay eficiencia en la medida que se mejora la eficiencia en el uso de los factores de la producción existirá la posibilidad de aumentar los ingresos del capital y las remuneraciones para los trabajadores ya que es necesario conocer el comportamiento de la productividad de ambos factores.

El cuestionamiento es ¿Cómo incide de la satisfacción laboral en el incremento de la productividad en las organizaciones?, de ahí que el propósito del presente artículo es determinar los factores de satisfacción laboral que inciden en la productividad organizacional.

\section{Satisfacción laboral y la productividad}

Las principales teorías sobre la satisfacción laboral surgieron a mediados del siglo pasado, permitiendo profundizar sobre los principales factores motivacionales que han sido un punto de partida para las nuevas y futuras teorías motivacionales, la teoría más relevante en este punto es la teoría de Abraham Maslow (Chiavenato, 2000), afirma que existe una disposición del trabajador hacia el desarrollo y maduración personal, esto corresponde a una jerarquía que es desde una satisfacción de necesidades básicas hasta una de necesidades secundarias. Cuando quedan satisfechas las necesidades fisiológicas básicas a través de una remuneración, dejan de ser atractivas para el trabajador ya que sentirá otras necesidades cómo es el reconocimiento o la autorrealización, y se une la confianza, creatividad, respeto, toma de decisiones, siendo una constante búsqueda de un bienestar emocional que impulsa al trabajador a conseguir ese propósito, lo cual se puede lograr individualmente o en trabajado en equipo.

La satisfacción- productividad puede resumirse en la afirmación de que un trabajador feliz es un trabajador productivo. Investigaciones indican que si hay una relación positiva entre la satisfacción y la productividad. La relación es más fuerte si el comportamiento del trabajador no está limitado o controlado por factores externos. La productividad de un empleado en puestos a ritmo de máquina está mucho más influida por la velocidad de la máquina que por su nivel de satisfacción. Por su parte (Newstrom, 2003) define que, los empleados se sumergen en su trabajo, al invertir tiempo y energía en él y lo consideran parte central de su vida. Contar con un trabajo que obtiene sentido y llevarlo a cabo satisfactoriamente son aspectos importantes de ellos mismos, lo cual ayu da a explicar el efecto traumático que la pérdida del trabajo tiene en las necesidades de autoestima.

También, la literatura explica que los colaboradores dedicados creen en la ética laboral, tienen necesidades de crecimiento altas y disfrutan de la participación en la toma de decisiones. Pocas veces llegan tarde o faltan, están dispuestos a trabajar largas jornadas e intentan lograr un rendimiento alto.

Así, el problema de la satisfacción laboral proviene a finales del siglo XIX los dueños de las organizaciones se preocuparon por condiciones de satisfacción de los empleados ligadas a los resultados, los empresarios

\footnotetext{
* Ventaja de la felicidad
} 
habían logrado riquezas y posición mediante el trabajo de otros, estaban obligados a asumirsus responsabilidades, no solo a la economía, sino frente al bienestar individual y colectivo de sus trabajadores (Barley \& Kunda, 1992), entonces este bienestar se centraba en establecer programas sociales para mejorar condiciones morales y mentales del empleado que cambiaran o mejoraran los aspectos laborales.

Con el surgimiento de la teoría de las relaciones humanas, la investigación de la eficiencia lleva a los empresarios al desarrollo de programas que trascienden la moralidad, para dar paso a acciones de beneficencia como prestaciones, vacaciones, permisos por enfermedad, servicios de salud y fondos de pensiones que aumentan la satisfacción del trabajador y su rendimiento.

Después de la segunda guerra mundial se traslada la atención a la búsqueda de estrategias para un incremento de lealtad, la motivación y hacia los sistemas de compensación, decisiones de enriquecimiento de trabajo y participación. En los años setenta, el concepto de satisfacción laboral empieza a ligarse más con la calidad de vida laboral y productividad en respuesta a la creciente preocupación en torno a la naturaleza poco recompensable del trabajo, lo que evidencia una decreciente satisfacción con el empleo (Freemont E. \& James E., 1990). Es decir, se trasciende de un enfoque asistencialista de dar cosas, hacia una propuesta orientada hacia un desarrollo humano integral dentro de la organización.

\section{Niveles de satisfacción laboral}

(Bain, 2015), menciona dos tipos de niveles de análisis en la satisfacción laboral.

1) Satisfacción general. Como indicador promedio que el colaborador percibe frente a las distintas etapas de su trabajo.

2) Satisfacción por facetas. Es el grado mayor o menor de satisfacción frente a aspectos específicos de su trabajo, tales como; reconocimiento, condiciones laborales, supervisión recibida, beneficios, políticas de la empresa, entre otras. En este punto, la satisfacción está relacionada al clima organizacional de la empresa y al desempeño laboral, el cual varía de un grupo a otro y se relacionan con diversas variables que, de acuerdo con este planteamiento gira alrededor de la edad, el nivel ocupacional y el tamaño de la organización.

Es decir, a medida que los trabajadores envejecen podría estar un poco más satisfechos con su trabajo, tiempo después, su satisfacción disminuye, porque son menos frecuentes las promociones y se enfrentan a la jubilación. Respecto a los trabajadores con nivel ocupacional más alto, tienden a estar más satisfechos con su trabajo, ya que es usual que estén mejor pagados, obtengan mejores condiciones de trabajo, además que sus puestos les permite aprovechar plenamente sus capacidades.

\section{Materiales y métodos}

Es una investigacion con enfoque cuantitativo inicia con una descripción, ya que se detalla aquellas formas importantes de cómo es la satisfacción laboral en los trabajadores de las empresas del sector manufacturero, se estableció el cálcu lo para medir el grado de correlación entre la satisfacción laboral y la productividad.

La muestra estuvo conformada por 60 trabajadores(as) de 3 empresas medianas del sector manufacturero textil localizadas en el municipio de Tepeji del Rio, del Estado de Hidalgo. El criterio para seleccionar la muestra fue la disponibilidad de participar en el estudio. Se integra con hombres y mujeres cuya edad oscila entre 20 y 55 años (ver tabla 1 y 2 ).

Tabla 1. Género de los participantes

\begin{tabular}{|l|l|}
\hline Género & Número \\
\hline Femenino & 11 \\
\hline Masculino & 49 \\
\hline Total & $\mathbf{6 0}$ \\
\hline
\end{tabular}

Tabla 2. Edad de participantes

\begin{tabular}{|l|l|}
\hline Edad/años & Número \\
\hline 20 a 35 & 16 \\
\hline 36 a 45 & 19 \\
\hline 46 a 55 & 25 \\
\hline Total & $\mathbf{6 0}$ \\
\hline
\end{tabular}

Para medir la satisfacción laboral se utilizó un cuestionario que integra 5 factores y 20 ítems. Las dimensiones que evalúan son:

Logro: Se refiere a los sentimientos de deber cumplido, resultados o rendimientos y metas alcanzadas.

Reconocimiento: Es aquel tipo de reconocimiento o elogios recibidos por su trabajo, sus jefes, compañeros, subordinados

Trabajo en Sí: Se refiere a cómo percibe su trabajo, le es atractivo, desafiante, variado, creativo, etc.

Responsabilidad: Aquí se abarca al nivel de responsabilidad de su propio trabajo y del de otros, así como el nivel de importancia que le brinda.

Promoción: Se refiere a la posibilidad de ascenso, formación de cargo de la empresa

Los ítems son de escala tipo Likert que tiene como alternativa de respuesta: a veces, generalmente y casi siempre. El cuestionario fue aplicado in situ a los trabajadores de las distintas empresas. 
Para medir la productividad se empleó un cuestionanio con 20 ítems dirigidos a los jefes de área, el objetivo fue evaluar a los trabajadores que tienen a su cargo en el desempeño de sus funciones, por ejemplo: ¿la empresa brinda facilidades para mejorar la productividad? Con opción de respuesta a) de acuerdo b) ni de acuerdo ni en desacuerdo c) en desacuerdo ¿La supervisión permite lograr mayor productividad en sus trabajadores? ¿La productividad le ha permitido captar más clientes? ¿Considera que en el área técnica la productividad es óptima? ¿Considera que en el área administrativa la productividad es óptima? Con opción de respuesta a) a veces, b) generalmente c) casi siempre.

\section{Resultados}

En primer término, se presenta la caracterización de los factores de satisfacción laboral.

Respecto a la satisfacción de los trabajadores con las metas alcanzadas, ésta tiene un alto grado de aceptación, lo cual permite identificar que el personal logra cumplir con las metas que la organización plantea (Grafica 1).

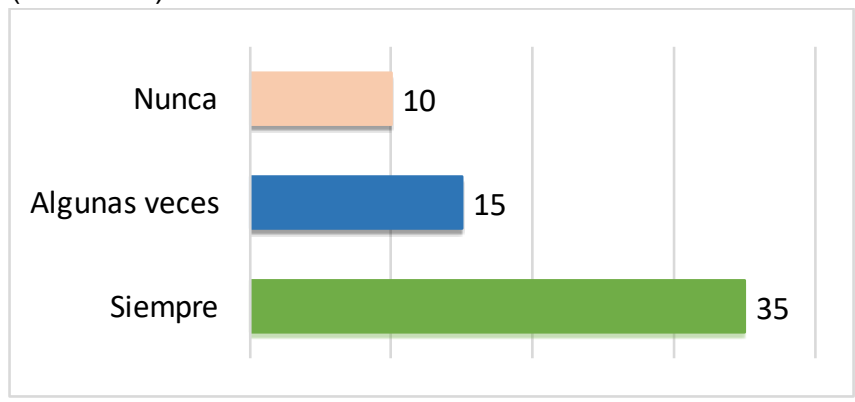

Grafica 1. Satisfacción con las metas alcanzadas. A partir de las respuestas de los participantes.

Al obtener una buena disposición y trabajo en equipo, se alcanzan las metas deseadas, contribuyendo al logro de objetivos y reconocimiento del trabajador por jefes inmediatos (Grafica 2).

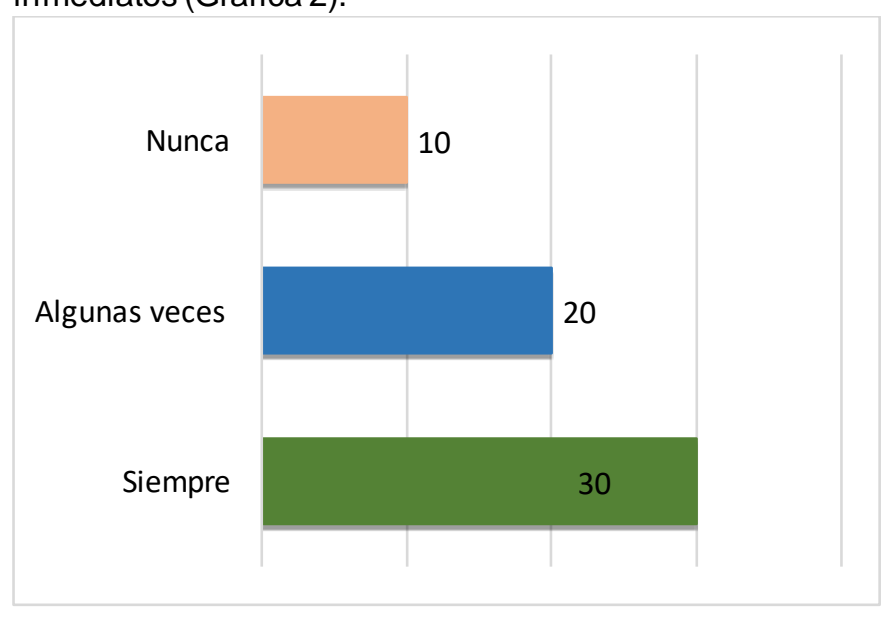

Grafica 2. Trabajo reconocido por la empresa, logrando los objetivos y metas. A partir de las respuestas de los participantes.

Al realizar el trabajo de forma correcta, la satisfacción está presente al coordinar los equipos de trabajo, tareas y relaciones laborales.

En la siguiente grafica 3, los trabajadores disfrutan de su trabajo realizado con sus compañeros, dentro de diferentes áreas de la empresa.

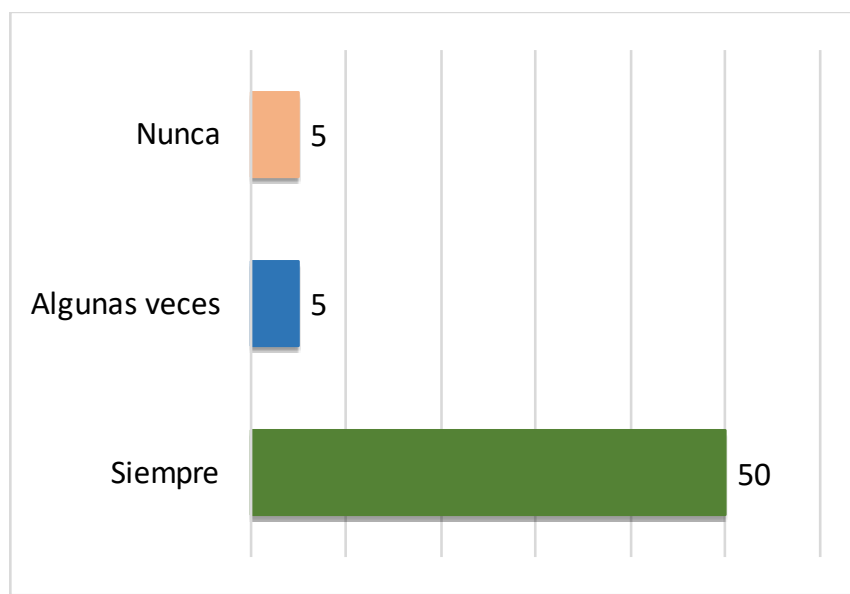

Grafica 3. Trabajo alcanzado por compañeros de otras áreas siendo satisfactorio. A partir de las respuestas obtenidas por los colaboradores.

Al tener la información correcta los trabajadores realizan en forma correcta su trabajo sintiéndose parte de la organización.

Respecto a cómo los colaboradores están dispuestos asumir nuevas responsabilidades en su área de trabajo se observa un resultado positivo (Ver grafica 4).

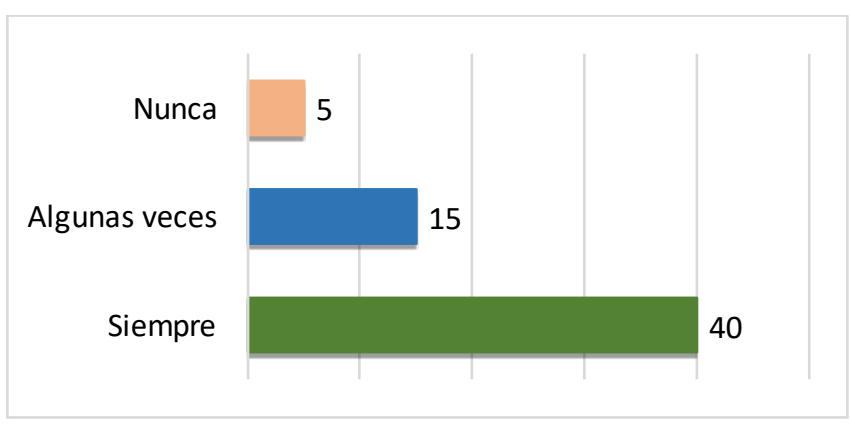

Grafica 4. Disponibilidad de asumir nuevas responsabilidades. A partir de las respuestas obtenidas de los participantes.

En la siguiente grafica se observa que, al lograr las metas establecidas se puede ascender a otro puesto dentro de 
la organización, teniendo la oportunidad de desarrollar una carrera laboral dentro de la empresa (Ver grafica 5).

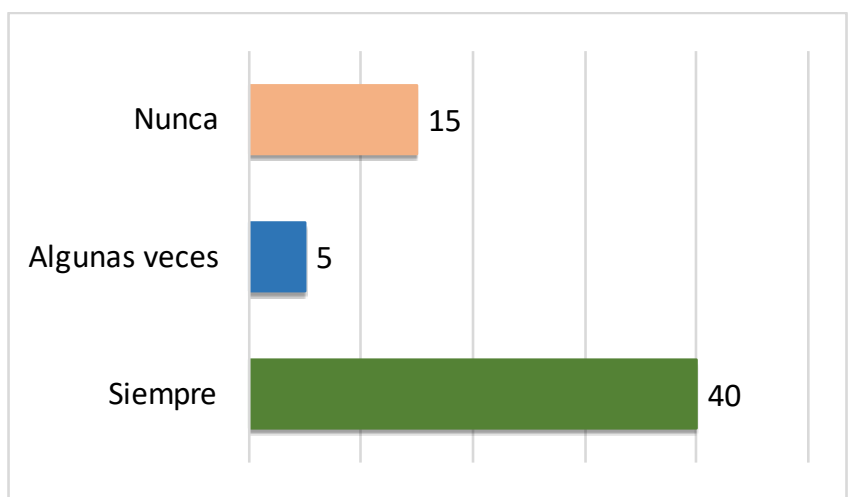

Grafica 5. Logro de metas y ascensos ganados. A partir de las respuestas obtenidas de los participantes.

Ahora, se presentan los factores que inciden en la productividad organizacional (Tabla 3). Para ello, se realiza el cálculo de correlación entre la satisfacción laboral y la productividad.

Tabla 3. Resultados de correlación

\begin{tabular}{|l|c|c|c|c|c|c|}
\hline Test & $\boldsymbol{x}$ & $\boldsymbol{y}$ & $\boldsymbol{x} \mathbf{2}$ & $\boldsymbol{y} \mathbf{2}$ & $\boldsymbol{x}, \boldsymbol{y}$ & $\boldsymbol{r} \mathbf{2}$ \\
\hline $\begin{array}{l}\text { Escala } \\
\text { likert }\end{array}$ & 60 & 60 & 150 & 49 & 0,039 & 0,023 \\
\hline
\end{tabular}

Fuente. Elaboración del autor con datos de encuesta.

Con un nivel de confianza $0.05 \%$ en donde el $95 \%$ equivale a $z=1.96$

\section{Como se puede observar, la correlación es de 0.039 , por lo que no existe correlación entre las variables satisfacción laboral $y$ productividad, para el presente estudio, cada variable es independiente, lo que significa que los trabajadores están satisfechos con su trabajo, sin embargo; esto no impacta o afecta la productividad}

\section{Discusión}

Al plantear el objetivo de realizar un estudio, se encontró que las empresas estudiadas mantienen condiciones de trabajo que propician la satisfacción laboral y además presentan productividad desde la perspectiva de sus jefes inmediatos. Queda claro que estadísticamente las percepciones de los trabajadores son independientes de la productividad en su ámbito laboral.

\footnotetext{
* Es un índice multilateral que puede ser usado para comparar la productividad (tecnología de producción y eficiencia) de diversas economías
}

Por su parte, los resultados del mercado de la industria textil indican que en los últimos 10 no ha mejorado su productividad. El índice de Malmquist ${ }^{*}$, que refleja las variaciones en la productividad de un periodo a otro, se mantuvo constante durante todo el ciclo con leves variaciones poco significativas. La principal causa se atribuye al aspecto tecnológico, este resultado no se ha podido recuperar lo que significa que, las empresas necesitaron más recursos para seguir produciendo lo mismo, no han invertido en mejorar sus prácticas administrativas, comerciales o tecnológicas, o bien sus inversiones. Ante este panorama y en contraposición con resultados a los obtenidos en esta investigación, el cambio técnico no ha contribuido a mejorar la productividad.

\section{Conclusiones}

Al aplicar los dos instrumentos, para evaluar el nivel de satisfacción laboral y productividad, se probaron elementos teóricos y sus respectivos indicadores, que comparan el tema de influencia de satisfacción laboral en la productividad, en tres empresas medianas del sector manufacturero textil localizadas en el municipio de Tepeji del Rio, del Estado de Hidalgo.

Se ha podido identificar en este momento, la percepción y expectativas de los trabajadores respecto a su ámbito laboral. Queda claro que la pretensión de las expectativas que tenga cada una de las partes, es decir empresa empleado variará de organización a organización, de persona a persona de periodo a periodo.

De acuerdo con los resultados obtenidos, se puede testificar que es importante para los trabajadores que los jefes den un buen seguimiento a su desempeño laboral, que les brinden una adecuada retroalimentación, que evalúen el cumplimiento de metas propuestas y que identifiquen constantemente los logros. Es menester, efectuar mediciones de satisfacción laboral periódicamente, para mantener información actualizada que contribuya a conocer cuáles son las necesidades del equipo que integran.

\section{Referencias}

[1] Ramirez, A. (2014). la felicidad es un excelente negocio. Una empresa con colaboradores felices, es un lugar en donde hay mayor compromiso y productividad. Emprende, 22-33. 
[2] Hernández Laos, E. (2002). La productividad en México. Origen y distribución, 1960-2002. Economiaunam,9-15.

[3] Andrew, O. (Octubre de 2010). Health, Happiness and productivity. Obtenido de Universidad de Warwick:

https://warwick.ac.uk/newsandevents/knowledge/socialscience/productivit y

[4] INEGI. (2015). Cálculo de los indices de productividad laboral. Instituto Nacionalde Esta dística y Geografía, 1-82.

[5] Chiavenato, I. (2000). Bogota Colombia: Mc Graw Hill

[6] Newstrom, D. (2003). Comportamiento humano en el trabajo. Mèxico: McGraw-Hill.

[7] Barley, S., \& Kunda, G. (1992). Escructura y diseño vs. leasltad y sentimiento olea das de ideologias racionales y normativas de controln en el discurso gerencial. Redalyc, 142.

[8] Fremont E., K., \& James E., R. (1990). Administración en las organizaciones (enfoque de sistemas y de contigencias). Mèxico, DF.: Mc Graw Hill.

[9] Bain, D. (2015). Productividad, la soluciòn de los problemas de la empresa en Mèxico. Mèxico: Grijalbo. 